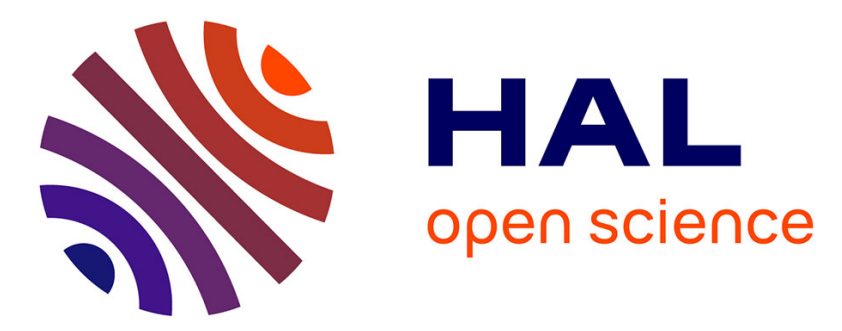

\title{
Extraction of velocity time series with an optimal temporal sampling from displacement observation networks
}

Laurane Charrier, Yajing Yan, Elise Colin Koeniguer, Silvan Leinss, Emmanuel Trouve

\section{To cite this version:}

Laurane Charrier, Yajing Yan, Elise Colin Koeniguer, Silvan Leinss, Emmanuel Trouve. Extraction of velocity time series with an optimal temporal sampling from displacement observation networks. IEEE Transactions on Geoscience and Remote Sensing, 2022, 60, pp.1-10. 10.1109/TGRS.2021.3128289 . hal-03436678

\section{HAL Id: hal-03436678 \\ https://hal.science/hal-03436678}

Submitted on 19 Nov 2021

HAL is a multi-disciplinary open access archive for the deposit and dissemination of scientific research documents, whether they are published or not. The documents may come from teaching and research institutions in France or abroad, or from public or private research centers.
L'archive ouverte pluridisciplinaire HAL, est destinée au dépôt et à la diffusion de documents scientifiques de niveau recherche, publiés ou non, émanant des établissements d'enseignement et de recherche français ou étrangers, des laboratoires publics ou privés. 


\title{
Extraction of velocity time series with an optimal temporal sampling from displacement observation networks
}

\author{
Laurane Charrier, Student Member, IEEE, Yajing Yan, Member, IEEE, Elise Colin Koeniguer, Silvan \\ Leinss, Senior Member, IEEE, and Emmanuel Trouvé, Senior Member, IEEE
}

\begin{abstract}
Today, more and more velocity observations are available online or on-demand. However, this amount of data is complex to analyze since velocity observations span different temporal baselines. Velocities obtained from a small temporal baseline are close to the derivative of the displacement but are more likely to be contaminated by noise. Velocities obtained from a long temporal baseline approximate the mean velocity between two dates but can be affected by temporal decorrelation. Having short and long temporal baselines provides a data redundancy that needs to be properly considered. In this article, we propose a method that aims to extract short-term velocity time series with a regular temporal sampling from all available displacement observations. The proposed method relies on a temporal inversion based on an improved temporal closure of the displacement observation network. Two criteria are proposed to determine the optimal temporal sampling to study short-term variations. To take the unequal data uncertainty into account, the temporal inversion is done by an Iterative Reweighted Least Square using a well-established weighting function, without preprocessing. The proposed method results in velocity time series with an optimal temporal sampling, an improved temporal coverage, reduced uncertainty and no redundancy. The studied area is the Kyagar glacier, in the North of the Karakoram range which is characterized by strong velocity variations originated from a glacier surge and additional seasonal variability.
\end{abstract}

Index Terms-time series, velocity, displacement, glacier, extraction, fusion, short-term variations, temporal closure network, homogenization, inversion

\section{INTRODUCTION}

$\mathbf{V}$ Elocity maps are necessary to precisely monitor ice dynamics, to infer sub-glacial processes and/or ocean forcing, and to derive other products such as mass-balance or strain rates when the amount of data is sufficient. Nowadays, many ice velocity observations derived from pairs of satellite images are available online [1]-[3] or on-demand [4]. This amount of data is complex to analyze since the velocity observations span different temporal baselines. Velocities covering small temporal baselines are close to the temporal derivative of the displacement, whereas long temporal baseline velocities approximate the mean velocity over the considered period.

L. Charrier, Y. Yan, S. Leinss, Emmanuel Trouvé are with the Laboratoire d'Informatique, Systèmes, Traitement de l'Information et de la Connaissance (LISTIC), Polytech Annecy-Chambéry, Université Savoie Mont Blanc, B.P. 80439, 74944 Annecy le Vieux Cedex FRANCE (e-mail: laurane.charrier@univ-smb.fr; yajing.yan@univ-smb.fr; silvan.leinss@univsmb.fr; emmanuel.trouve@univ-smb.fr).

E. C. Koeniguer is with ONERA, Département Traitement de l'Information et Systèmes, Université Paris-Saclay, Palaiseau, 91123, France (e-mail: elise.koeniguer@onera.fr)
Moreover, the uncertainty of velocity measurements differs. In the case of small temporal baselines, the noise of velocity can be larger than the velocity magnitude due to the small displacement magnitude, while in the case of long temporal baselines, the uncertainty mainly results from the surface changes between image acquisitions, which induces temporal decorrelation.

This is why some authors performed velocity data analysis by picking small or long temporal baselines measurements depending on their interest (e.g. small temporal baselines for intra-annual or long temporal baselines for inter-annual studies)[5], [6]. This process implies having enough reliable data, which cannot always be possible, especially in mountain areas. Therefore, other authors suggested integrating a regression function to fit displacement measurements [7] which requires a priori knowledge or assumptions on the displacement behavior.

In this paper, we propose a temporal inversion approach based on the temporal closure of the displacement observation network. The idea is inspired by the Small BAseline Subset (SBAS) approach [8]-[10], originally developed for Interferometric Synthetic-Aperture Radar (InSAR) time series. The SBAS approach uses interferograms produced by image pairs spanning small temporal and geometrical baselines to minimize temporal and geometrical decorrelation. Later, this approach has been applied to offset-tracking displacement measurements of SAR images [11]-[13] and optical images [14]. The SBAS approach has mostly been applied to retrieve common reference time series, i.e., series of displacements computed between a reference date and other dates. However, this approach can be adapted to time series of displacements between consecutive dates, here called leap frog time series similar to [15]. The latter is especially interesting for fast decorrelating targets such as glaciers.

In most papers deploying SBAS like approaches [8]-[14], [16], authors performed a preprocessing to select the data and to reject outliers before the inversion. They included only data with a quality of the correlation for offset-tracking [11][14] and coherence for InSAR [8]-[10] larger than a given threshold. However, this preprocessing requires that a reliable data quality indicator is available and threshold values are properly defined. Still, taking the data uncertainty into account universally remains an open issue.

Another important issue concerns the appropriate temporal baseline of the leap frog time series, namely temporal sampling in this article. Given the coverage frequency of 12 
days of the Sentinel-1 A/B satellites over the Himalayas, the temporal sampling can be multiples of 12 days. Depending on the data quality, the displacement behavior, and the research objective, the question arises if there exists an optimal temporal sampling? If so, how can we determine this optimal temporal sampling? Furthermore, when the chosen temporal sampling is different from the satellite coverage frequency, it happens that some available displacement measurements (i.e. whose start date or end date are not contained in the leap frog time series dates) are useless because of the lack of direct link in the temporal closure formulation. What can be done to make use of as many as possible available displacement measurements regardless of their temporal baselines? To the best of our knowledge, these issues have not yet been reported in the literature.

In this article, we aim to extract, from all available displacement observations, leap frog velocity time series of appropriate temporal sampling with less noise and complete temporal coverage. Main efforts are devoted to the aforementioned open issues. First, an improvement of the classical temporal closure formulation is proposed to make use of as many as possible of the available displacement measurements. Second, the temporal sampling length is jointly analyzed with the signal-to-noise ratio of the retrieved velocity time series. To discuss what can be the optimal temporal sampling, two criteria are defined: the Root Mean Square Error (RMSE) on stable ground and the Velocity Vector Coherence (VVC) on moving areas. Finally, data quality is taken into account using an Iterative Reweighted Least Square (IRLS) and a well-established weighting function, without the need of a preliminary selection of data. The proposed method is applied to the Kyagar glacier, in the North of the Karakoram range.

\section{STUDY AREA AND DATA}

The study area is the Kyagar (Keyajir) glacier, a polythermal glacier that showed repeated glacier surges in the past. It is situated in the North of the Karakoram range, in the HindukuschKarakorum-Himalaya-Mountain chain. The terminus of Kyagar is located at 4750 m.a.s.l., whereas its accumulation part extends upwards to almost 7000 m.a.s.l. The total area covered by the glacier is about $100 \mathrm{~km}^{2}$. Three upper glacier tributaries converge to form an $8 \mathrm{~km}$ long and $1.5 \mathrm{~km}$ wide glacier tongue covered by huge ice pinnacles [17]. Besides, the glacier sometimes dams the river of the Upper Shaksgam Valley, forming an ice-dammed lake. The lake impounded behind this ice dam is known to fill and empty repeatedly [18]-[20]. The drainage of the lake is sometimes responsible for Glacier Lake Outburst Floods (GLOF). The latest GLOF was observed on August 10, 2018, with a flood discharge of $1570 \mathrm{~m}^{3} / \mathrm{s}$ [19]. A surge of this glacier has happened from May 2014 to March 2016, leading to another GLOF in July 2015. According to [21], velocities have appeared to evolve in a manner consistent with a hydrologically controlled surged mechanism.

The considered data-set contains displacement observations computed from Sentinel-1 ortho-rectified ascending images acquired between October 14, 2014, and June 30, 2020, on the Kyagar glacier. The displacements are generated by cross

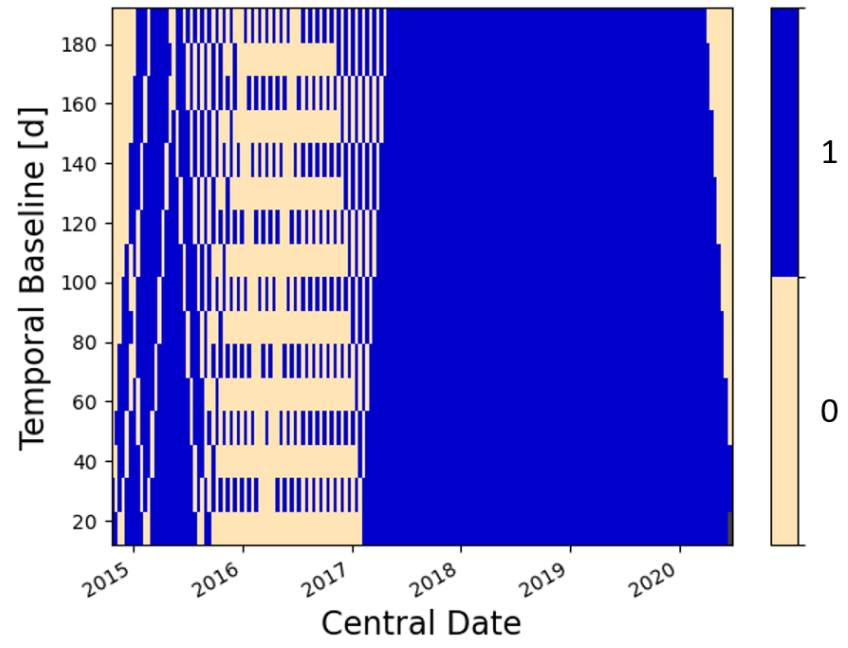

Fig. 1. Available velocity observations. The $x$-axis is the central date of the velocity observations and $y$-axis their temporal baseline. On the $\mathrm{x}$ and $\mathrm{y}$-axis, the center of each bin is 12 days apart. The values are 0 (when no velocity observation was measured for the corresponding central date and temporal baseline) or 1 .

correlation with a correlation window size of $64 \times 64$ pixels [21]. The horizontal posting of velocity maps is $200 \mathrm{~m}$. The temporal baselines range from 12 to 192 days. A quality indicator is given by averaging four factors: 1) The value of the Normalized Cross-Correlation (NCC) peak, 2) A measure of the shape of this peak (equal to 1 if there is a clear maximum and to 0 if there are multiple side maxima), 3) A measure which analyzes how broad the NCC peak is (if the peak is sharp, this factor will be equal to 1 while multiple scattered peaks will reduce the value by the 1/average distance of the peaks) 4) A measure of the distance between the NCC peak calculated in the low and in the high-frequency components of the spectrum (equal to 1 if both show the same location).

It is worth mentioning that Sentinel-1 frequency coverage was not constant over the considered region from 2014 to 2020. In particular, between October 9, 2015 and July 7, 2017, the time interval between two images was 24 days instead of 12 days, as shown in Figure 1.

\section{METHOD}

\section{A. Improved temporal closure of displacement observation network}

The proposed method is applied on a pixel-by-pixel basis on the set of $n$ displacement observations $d_{t_{i}, t_{j}}$. Each displacement $d_{t_{i}, t_{j}}$ have a temporal baseline $t_{j}-t_{i}$ between the acquisition times $t_{i}$ and $t_{j}$. This temporal baseline is a multiple of the satellite coverage frequency $\Delta t$. The key principle relies on making use of the redundancy of the displacement observation network to build a leap frog displacement time series which respects the temporal closure constraint. It uses all the available displacement observations to obtain leap frog displacement time series with a constant temporal sampling $\Delta \tau . \Delta \tau$ is a multiples of $\Delta t$ and can be chosen by the enduser. 
a)

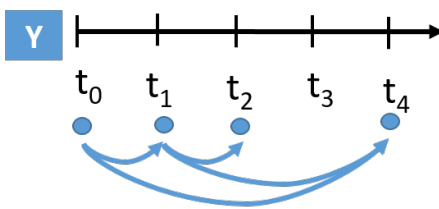

$x$

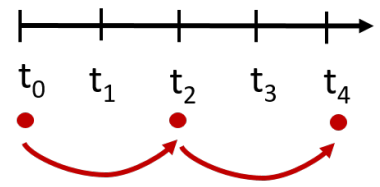

$\mathrm{X}=\left[\begin{array}{l}\hat{d}_{t_{0}, t_{2}} \\ \hat{d}_{t_{2}, t_{4}}\end{array}\right]$

a) b)

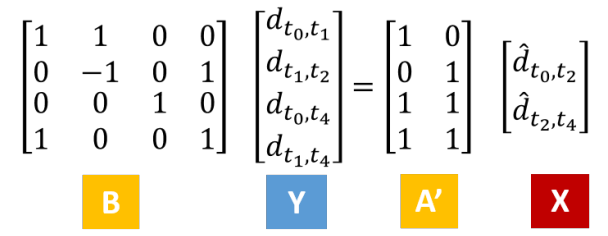

Fig. 2. a) Illustration of the classical temporal closure of the displacement observation network b) Illustration of the improved temporal closure of the displacement observation network. The vector $Y$ corresponds to the displacement observations. The vector $X$ stands for the leap frog displacement time series. $A$ is the design matrix linked $X$ and $Y$ in the classical temporal closure. $A^{\prime}$ and $B$ are the design matrix linking $X$ and $Y$ in the improved temporal closure.

The classical temporal closure formulation is $Y=A X$ where the vector $Y$ contains all $n$ displacements observation, $X$ represents the vector of $p$ output displacements $\hat{d}_{t_{k}, t_{k}+\Delta \tau}$, and $A$ is a design matrix of dimension $n \times p$ linking $X$ with $Y$. With this design, a part of the displacement observations $d_{t_{i}, t_{j}}$ in $Y$ can be directly linked to a sum of displacements $\hat{d}_{t_{k}, t_{k}+\Delta \tau}$ from $X$. These linear equations are represented by rows of $A$. For instance, in Figure 2, the third row establishes the link between the displacement observation $d_{t_{0}, t_{4}}$ and the leap frog time series displacements $\hat{d}_{t_{0}, t_{2}}$ and $\hat{d}_{t_{2}, t_{4}}: d_{t_{0}, t_{4}}=$ $\hat{d}_{t_{0}, t_{2}}+\hat{d}_{t_{2}, t_{4}}$. However, if one of the date $t_{i}$ or $t_{j}$ of the displacement observation is not included in the list of dates of the leap frog time series $\mathrm{T}=\left[t_{0}, t_{1 * \Delta \tau}, t_{2 * \Delta \tau}, \ldots, t_{p * \Delta \tau}\right]$ (with $t_{0}$ the first date of acquisition inside the data-set), no direct relation can be established with the classical formulation. Hence, this displacement observation cannot be used resulting in a row of zeros in A. For example, in Figure 2a), three displacement observations are not used: $d_{t_{0}, t_{1}}, d_{t_{1}, t_{2}}$ and $d_{t_{1}, t_{4}}$ because $t_{1}$ is not contained in $T=\left[t_{0}, t_{2}, t_{4}\right]$. The corresponding rows contain only 0 . Some information is lost.

Therefore, we propose an improved temporal closure formulation by adding a design matrix $B$ that permits to replace the rows in $A$ containing only zeros by linking the leap frog displacements $d_{t_{k}, t_{k}+\Delta \tau}$ with combinations of observation displacements $d_{t_{i}, t_{j}}$. It makes it possible to link as many as possible of the available displacement observations in $Y$

with the displacements in $X$. The new system is $B Y=A^{\prime} X$ where $B$ is a design matrix of dimension $n^{\prime} \times n$ and $A^{\prime}$ is the design matrix of dimension $n^{\prime} \times p$, with $n^{\prime}$ the number of displacement combinations as specified below. For example, in the Figure $2 \mathrm{a}$ ), there is no relation between $d_{t_{0}, t_{1}}$ and the leap frog displacements, because the date $t_{1}$ is not contained in T. However, it can be established that $d_{t_{0}, t_{1}}+d_{t_{1}, t_{2}}=\hat{d}_{t_{0}, t_{2}}$ as illustrated in Figure $2 b$ ). In this way, solving the equation $B Y=A^{\prime} X$ leads to compute the minimized square distance between the displacements $\hat{d}_{t_{0}, t_{2}}$ inferred from all possible temporal closures.

The matrices $A^{\prime}$ and $B$ are designed so that each row of the resulting system $B Y=A^{\prime} X$ represents a linear equation linking a displacement observation $d_{t_{i}, t_{j}}$, or a combination of displacement observations, with a sum of leap frog displacements $\hat{d}_{t_{k}, t_{k}+\Delta \tau}$. The following four cases need to be considered:

1) If the dates $t_{i}$ and $t_{j}$ are included in $\mathrm{T}$, the linear equation is:

$d_{t_{i}, t_{j}}=\sum_{k=i}^{j-\Delta \tau} \hat{d}_{t_{k}, t_{k+\Delta \tau}}$.

Hence, the row in $B$ contains only a 1 on the diagonal and the row in $A^{\prime}$ is simply filled with 1 to describe the sum of the corresponding leap frog displacements. This is equivalent to the classical method.

2) If $t_{i}$ is not in $T$, one of the following cases is done, with $\mu$ the smallest integer to obtain a displacement combination matching the leap frog displacements:

a) add the smallest preceeding displacement $d_{t_{i-\mu}, t_{i}}$ : $d_{t_{i-\mu}, t_{i}}+d_{t_{i}, t_{j}}=\sum_{k=i-\mu}^{j-\Delta \tau} \hat{d}_{t_{k}, t_{k+\Delta \tau}}$

b) subtract the smallest displacement starting at $t_{i}$, $d_{t_{i}, t_{i+\mu}}$ from $d_{t_{i}, t_{j}}$ :

$d_{t_{i}, t_{j}}-d_{t_{i}, t_{i}+\mu}=\sum_{k=i+\mu}^{j-\Delta \tau} \hat{d}_{t_{k}, t_{k+\Delta \tau}}$

3) If $t_{j}$ not in $\mathrm{T}$, one of the following cases is done:

a) add the smallest consecutive displacement $d_{t_{j}, t_{j+\mu}}$ : $d_{t_{i}, t_{j}}+d_{t_{j}, t_{j+\mu}}=\sum_{k=i}^{j+\mu-\Delta \tau} \hat{d}_{t_{k}, t_{k+\Delta \tau}}$

b) subtract the smallest displacement ending at $t_{j}$, $d_{t_{j-\mu}, t_{j}}$ from $d_{t_{i}, t_{j}}$ :

$d_{t_{i}, t_{j}}-d_{t_{j}-\mu, t_{j}}=\sum_{k=i}^{j-\mu-\Delta \tau} \hat{d}_{t_{k}, t_{k+\Delta \tau}}$

4) If $t_{i}$ and $t_{j}$ not in $\mathrm{T}$ : both 2) and 3) are done

Applying the above cases to each of the $n$ observed displacement results in $n$ rows of equation where $A^{\prime}$ and $B$ are filled in consequence with $1,-1$ and 0 . As we want to use every generated combination only once, redundant rows are removed. So, the number of rows in $A^{\prime}$ is $n^{\prime}<=n$.

\section{B. Iterative Reweighted Least Square inversion}

To solve the equation $B Y=A^{\prime} X$ on each pixel, an Iterative Reweighted Least Square (IRLS) approach is proposed. A 
regularization term on the discrete derivative of the leap frog velocities can be added as done in [14] assuming that ice velocities have a low temporal variability on a short time scale (i.e. with a small temporal sampling length). Therefore, the cost function to minimize is defined as:

$$
\arg \min \left(\left\|W\left(A^{\prime} X-B Y\right)\right\|^{2}+\lambda\|\Gamma X\|^{2}\right)
$$

where $W$ is a $n^{\prime} \times n^{\prime}$ matrix standing for the weight given to each value in $B Y, \lambda$ is a scaling constant and $\Gamma$ is a $p \times$ $p$ matrix representing the discrete derivative operator of the velocities. The diagonal element of $\Gamma$ are $\Gamma_{k, k}=1 / \Delta \tau$ and the element above the diagonal are $\Gamma_{k, k+1}=-1 / \Delta \tau$ with $\Delta \tau$ the temporal sampling.

The scaling constant $\lambda$ should be small enough to affect only values in $X$ weakly constrained by the inversion [14]. This is the case when a value in $X$ is related to only few displacement observations in the temporal closure network or when disconnected data subsets exist [10], [14]. This value is set to 1 for the considered data-set.

The solution is given by the equation (2). A Singular Value Decomposition (SVD) can also be used if the system is illposed (i.e. when the rank of $A$ is lower than $p$ ) [8]:

$$
\hat{X}=\left(A^{T} W A+\lambda \Gamma^{T} \Gamma\right)^{-1} A^{T} W B Y
$$

The proposed approach includes several iterations.

1) First iteration: A first Weighted Least Square (WLS) inversion is performed. The weight $W$ in equations (1) and (2) is initialized as $W^{0}$, a diagonal matrix of dimension $n^{\prime} \times$ $n^{\prime} . W^{0}$ contains the weights applied to each combination of displacement observations in $B Y$. For the considered dataset, $W^{0}$ is set according to the error propagation in $B Y$. The errors of the elements of $Y$ are approximated by the inverse, element wise, of the quality indicator vector $Q$. The errors are then propagated by multiplication with $|B|$, where $|\cdot|$ indicates the absolute values, element-wise, of $\mathrm{B}$. The weight $W^{0}$ is then set to the inverse, element wise, of the propagation errors, i.e., $W^{0}=\frac{1}{|B| \frac{1}{Q}}$. Note, that if no data quality indicator is available, $W^{0}$ can be initialized with the identity matrix.

2) Next iterations: Then, another least-square inversion is performed.

Since the data quality is often not accurately known, the residual of the first inversion provides complementary information on the consistency of the input displacement observations. It can be further used to refine the weight matrix $W$. The classical way consists of defining the weight as the inverse of the residual [14]. Here, a down-weight function inspired from [22] is used to be more robust to outliers.

First, internally-studentized residuals at iteration $u$ are defined as:

$$
Z^{u}=\frac{R^{u}}{\sigma \sqrt{1-H^{u}}}
$$

where $R^{u}$ is the residual vector of dimension $n^{\prime}$ (the difference between the reconstructed displacement observations computed with $A^{\prime} X$ and the original combinations of displacement observations $B Y$ ), $H^{u}$ the leverages vector (of dimension $n^{\prime}$ ), i.e. the diagonal elements of the hat matrix $A\left(A^{T} W A+\lambda \Gamma^{T} \Gamma\right)^{-1} A^{T} W, \quad \div$ the element wise division and $\sigma=\sqrt{\sum_{i=1}^{n^{\prime}} \frac{R_{i}^{u^{2}}}{\left(n^{\prime}-p\right)}}$ the standard deviation of residuals.

Then, the diagonal elements of the weight at iteration $u$ denoted $W^{u}=\operatorname{diag}\left(W_{1,1}^{u}, \ldots, W_{m, m}^{u}, . ., W_{n^{\prime}, n^{\prime}}^{u}\right)$ are updated as follow:

$$
W_{m, m}^{u}=\psi\left(\frac{1}{W_{m, m}^{0}} Z_{m}^{u}, c\right)
$$

where $\psi$, the Tukey's biweight function, which is a common down-weight function [22] robust to large outliers, is:

$$
\psi(z, c)=\left\{\begin{array}{l}
{\left[1-(z / c)^{2}\right]^{2},|z|<c} \\
0,|z|>c
\end{array}\right.
$$

where $c$ is a tuning constant which is usually set to 4.685, producing $95 \%$ efficiency at a normal distribution [23].

Then, the Weighted Least Square inversion described in this section (section III-B2) is further iterated by updating $W$ with the result from the previous iteration. The algorithm stops when mean $\left(\left|\hat{X}^{i}-\hat{X}^{i-1}\right|\right)<\delta$ where $\hat{X}^{i}$ corresponds to the results of a given iteration and $\hat{X}^{i-1}$ the results of the previous one. $\delta$ is a predefined threshold much smaller than the tracking accuracy of the displacements, here set to $0.001 \mathrm{~m}$.

Finally, each displacement in $\hat{X}$ is divided by the temporal sampling $\Delta \tau$ to obtain leap frog velocity time series in $\mathrm{m} / \mathrm{d}$.

On the one hand, this method tends to down-weight outliers which will have larger internally studentized-residuals and smaller weights. On the other hand, the regularization term helps to filter out unrealistic values when the inversion is weakly constrained by the observations.

\section{Uncertainty propagation}

The uncertainty of the resulting time series can be obtained theoretically by propagation through the inversion. The uncertainty is assumed to be random and independent for each displacement, hence the probability theory lead to propagate the diagonal covariance matrix of the error of displacement observations [22], [24] as:

$$
\Sigma_{\hat{X}}=\sigma_{0}^{2} N^{-1} A^{T} W B \Sigma B^{T}\left(N^{-1} A^{T} W\right)^{T}
$$

with $\Sigma_{\hat{X}}$ the covariance-matrix of the error of the leap frog time series obtained with the improved temporal closure of the displacements. $W$ is the vector which contains the weights obtained at the last iteration of the IRLS. $N$ is equal to $A^{T} W A+\lambda \Gamma^{T} \Gamma . \Sigma$ is the a-priori covariance-matrix of the error of displacement observations. If the a-priori covariancematrix of the error of displacement observations is sufficiently representative of the real covariance matrix, $\sigma_{0}^{2}$ is equal to 1. Otherwise, $\sigma_{0}^{2}$, estimated in equation 7 , scales the a-priori covariance-matrix of the error of displacement observations taking into account the values of the residuals.

$$
\sigma_{0}^{2}=\frac{R^{T} W R}{n_{e f f}^{\prime}-p}
$$


with $n_{\text {eff }}^{\prime}$ the number of effective observations after outlier removal based on the biweight function [22].

\section{TEMPORAL ANALYSIS}

\section{A. Time series with different temporal samplings}

Several temporal samplings could be chosen as output: $1 \Delta t, 2 \Delta t, 3 \Delta t, \ldots$ with $\Delta t$ the satellite coverage frequency, here of 12 days. The performance of the proposed approach using these different short-temporal samplings is assessed by computing two metrics for stable ground and fast-moving areas respectively.

On the stable ground, velocities should have a zero value. Therefore, the Root Mean Square Error (RMSE) of the velocity magnitude on the stable ground is computed:

$$
\operatorname{RMSE}=\operatorname{mean}_{(i, j) \in \omega_{1}} \sqrt{\frac{1}{N+1} \sum_{t=0}^{N}\|\vec{V}(i, j, t)\|^{2}}
$$

where $\vec{V}(i, j, t)$ stands for the velocity vector at time $t$ and pixel $(i, j), N+1$ is the number of velocity data at pixel $(i, j)$ over the considered period, $\omega_{1}$ corresponds to ice-free areas defined according to the Randolph Inventory V6.0 [25].

On glaciers, the flow direction on a point can be assumed to be roughly stable in time. Therefore, the temporal coherence of the flow direction is computed using the Velocity Vector Coherence (VVC) metric [26], [27] defined as:

$$
\mathrm{VVC}=\operatorname{mean}_{(i, j) \in \omega_{2}}\left\|\sum_{t=0}^{N} \frac{\vec{V}(i, j, t)}{\|\vec{V}(i, j, t)\|}\right\|
$$

where $\omega_{2}$ corresponds to the glacier parts where the magnitude of the mean velocity observations is higher than $0.2 \mathrm{~m} / \mathrm{d}$.

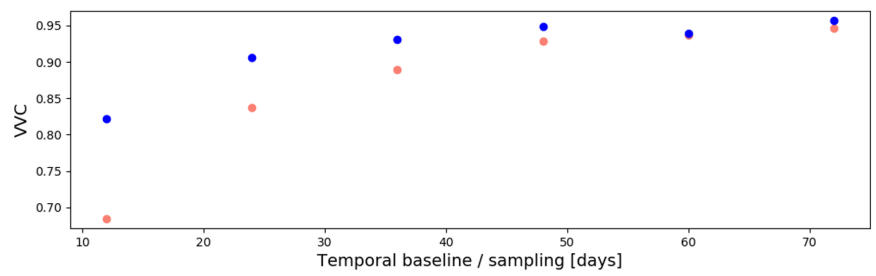

- Velocity observations - Leap Frog velocity time series

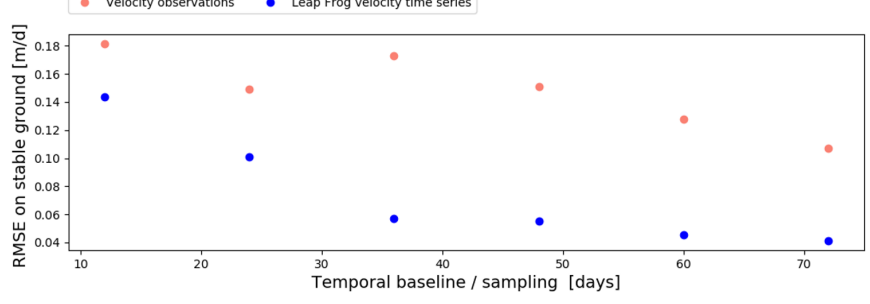

- Velocity observations - Leap Frog velocity time series

Fig. 3. Comparison of the VVC in moving areas where magnitude of the mean velocity observations is higher than $0.2 \mathrm{~m} / \mathrm{d}$ and of the RMSE on stable ground for different temporal sampling. The RMSE is performed on ice-free areas according to the Randolph Inventory V6.0 [25].

Figure 3 shows that for each temporal sampling and baseline, the VVC is higher, and the RMSE is lower for the leap frog velocity time series. This means that the inversion helps to increase the coherence of the velocity vectors and to reduce the uncertainty. Indeed, the RMSE on stable ground, which can be a proxy of the uncertainty, is decreased by $0.04 \mathrm{~m} / \mathrm{d}$, i.e. $22 \%$ for 12 -days temporal baselines to $0.12 \mathrm{~m} / \mathrm{d}$, i.e. $67 \%$ for 36-day temporal baselines.

Second, one can observe on the Figure 4 that even in the period where no velocities were measured with a temporal baseline of 12 and 36 days (grey rectangle in Figure 4), the IRLS inversion was able to retrieve 12 and 36 days velocities by using all the other velocity observations.

Therefore, the proposed method leads to reduced uncertainty and increased temporal coverage for any temporal sampling chosen as output.

\section{B. Time series with an optimal temporal sampling to study short-term variations}

But how to choose the length of this temporal sampling? Figure 3 highlights that the longer is the temporal sampling, the higher is the VVC, and the lower is the RMSE. This implies that the noise decreases and the coherence increases when the temporal sampling increases, as illustrated in Figure 4. The longer is the temporal sampling, the smoother is the leap frog time series, i.e., there is less noise but also less temporal resolution. Therefore, to study short-term variations of velocity, there is a need to find a compromise between the noise level of the velocity and the displacement behavior details as also emphasized in [4]. This means that velocities computed with the shortest temporal sampling (here 12 or 24 days) are not always the most appropriate to study short-term variations of velocities.

The VVC and the RMSE could be useful criteria to find the optimal temporal baseline and sampling. For the velocity observations (in red in Figure 3), the VVC seems to converge to 0.94 for a temporal baseline of 48 days. There is no further improvement with a longer temporal baseline. For 48-days velocity observations, the RMSE on the stable ground is 0.15 $\mathrm{m} / \mathrm{d}$. However, the observed velocity magnitude after 2017 is between 0.1 and $0.25 \mathrm{~m} / \mathrm{d}$ for the point presented in Figure 4 which means that the uncertainty is larger than $50 \%$ of the observed values after 2017. After inversion (in blue in Figure 3 ), the VVC reaches an asymptote for a temporal sampling of 36 days. The corresponding RMSE is $0.06 \mathrm{~m} / \mathrm{d}$. In conclusion, the VVC and RMSE can be used to select an optimal tempo$\mathrm{ral} / \mathrm{baseline}$. Moreover, the proposed inversion method allows the end-user selecting a smaller optimal temporal baseline with less uncertainty.

\section{Internally studentized-residual}

During the different iterations of the IRLS, the displacement observations are weighted by a combination of an a priori indicator of the data quality described in Section II and the internally studentized-residuals described in equation (3). In most cases, both weight indicators are consistent. However, the internally studentized residual provides complementary information which are of particular interest when the quality indicator is biased. Figure 5 shows that two encircled points have a data quality indicator around 0.4 in the original velocity 

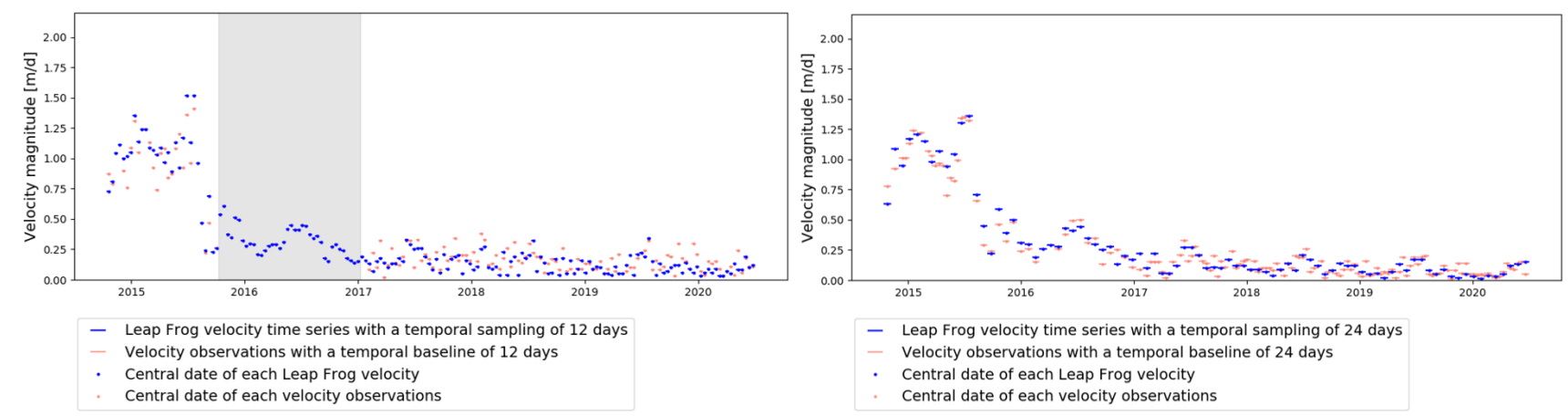

Velocity observations with a temporal baseline of 12 days

Central date of each velocity observation

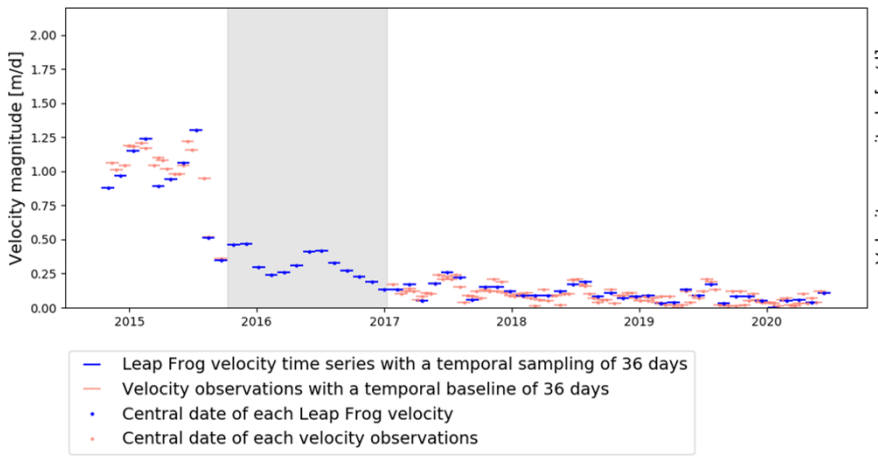

- Leap Frog velocity time series with a temporal sampling of 24 days Velocity observations with a temporal baseline of 24 days

Central date of each Leap Frog velocity Central date of each velocity observations

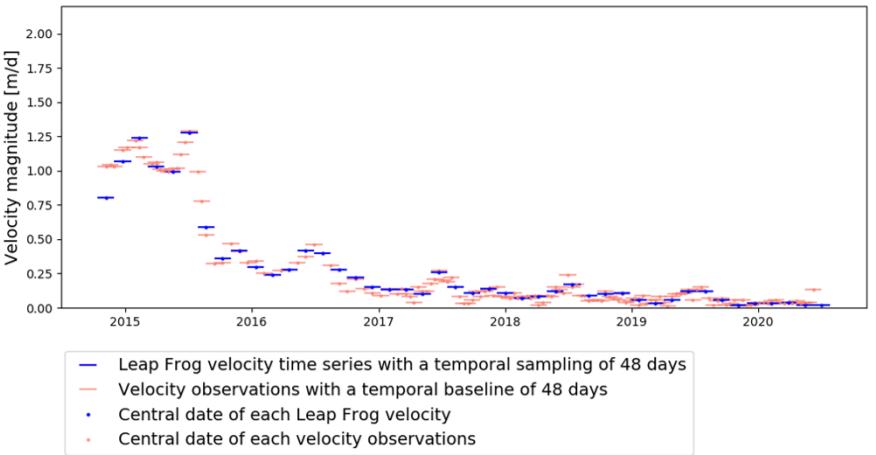

Fig. 4. Leap frog time series with different temporal sampling, compared with velocity observations of the same temporal baselines over a point situated near the Kyagar terminus at the coordinates (N35.67,E77.17). The blue and pink dots represent the central date of each leap frog velocity and velocity observation respectively. The blue and pink lines stand for the temporal baseline of each velocity observation and the temporal sampling of each leap frog velocity. The grey rectangle corresponds to the period where the Sentinel-1 images were acquired every 24 days instead of 12.
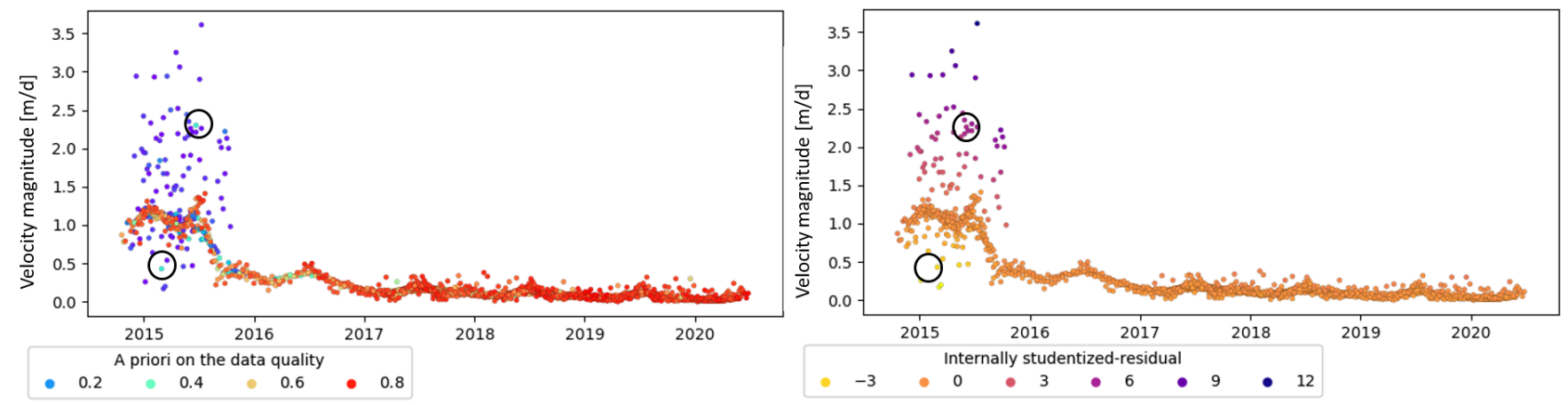

Fig. 5. Weights used for the inversion of velocities with a temporal sampling of 12 days on the point of coordinates (N35.67,E77.17). For visualization, the central date of each velocity observation data is represented only with a dot. On the left, the original velocity observations are colored with a priori data quality described in Section II. On the right, it is colored with internally studentized-residual computed after the last iteration of the IRLS. Two points with the data quality indicator higher than 0.4 but appearing to be outliers in the trend are encircled in black.

observations though these points stand out from the trend and are surrounded by lower quality data. These points appear to be outliers but still have relatively good data quality indicators. On the other hand, these two encircled points have the same internally-studentized residual as the surrounding points, which corresponds better to the reality after detailed inspection. The internally-studentized residuals rely on the difference between the displacement observations and the Least Square solution whereas the data quality indicator relies on the properties of the cross correlation function. Therefore, the internally-studentized residuals is a reliable way to account for data uncertainty. Note, however, that special attention still should be paid to internally-studentized residual values resulting from a weakly constrained IRLS, i.e., where a few displacement observations are available in the displacement observation network.

\section{Spatial ANALYSiS}

\section{A. Mean of velocity magnitude}

The mean of velocity observation magnitudes with a temporal baseline of 36 days is compared with the mean of velocity time series magnitudes with a temporal sampling of 36 days.

Figure 6 highlights that the velocity magnitudes are on average $0.09 \mathrm{~m} / \mathrm{d}$ higher after inversion than before over the 

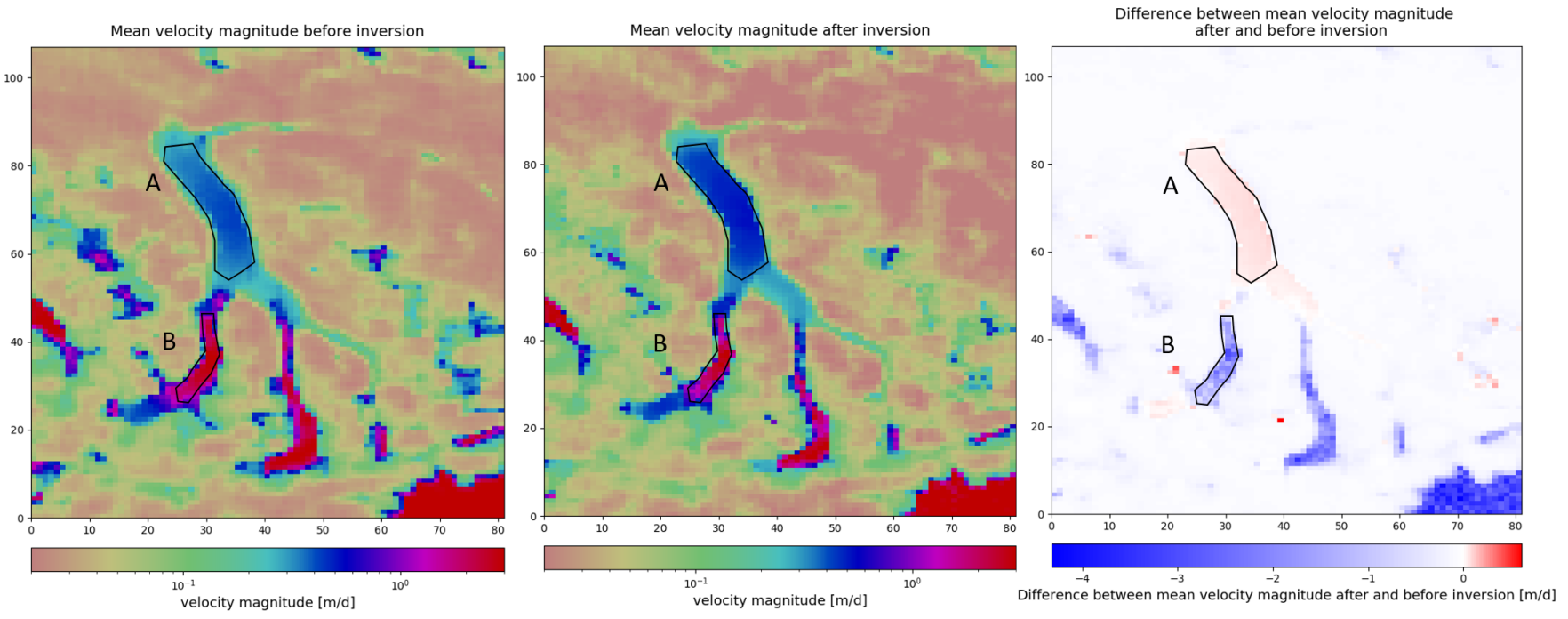

Fig. 6. Mean of velocity magnitude from October 14, 2014 to June 30, 2020 on each pixel for a temporal baseline and sampling of 36 days, before inversion on the left and after inversion on the middle. The sub-figure on the right highlights the difference between the mean velocity magnitude after and before inversion. The area A, corresponding to the glacier tongue, and the area B, corresponding to the lower part of the western tributaries are encircled for analysis.

glacier tongue (zone A) from 2014 to 2020. This represents an increase of $24 \%$ of the observed mean. This increase may be due to the lack of 36-day temporal baselines velocity observations in 2016 ( $c f$. Figure 1). Therefore, the same analysis is carried on data sets from January 7, 2017 to June 30, 2020. The increase in velocity is found to be $7 \%$ after inversion for this period. Hence, the lack of 36-day temporal baselines in 2016, a year where the mean of velocity magnitude is higher than the average, explains the underestimation of the mean velocity magnitude before inversion. This analysis highlights the importance of the temporal coverage of the velocity data to derive mean velocities.

\section{B. Spatio-temporal variations of short-term velocities}

The variation of the velocity magnitude is illustrated, in Figure 7, along with a longitudinal profile that goes from the upper part of the western tributary to the glacier tongue as represented in black in Figure 8. Over the glacier tongue, the main surge phase is visible from November 01, 2014, to August 16, 2015, consistently with observations on the temporal profile presented in Figure 4. The maximum of the 36-day velocity magnitudes is around $2 \mathrm{~m} / \mathrm{d}$ in November 2014. Then, the velocity magnitudes decrease during the winter to reach roughly $1.3 \mathrm{~m} / \mathrm{d}$ in April 2015. This is followed by an acceleration from May to July 2015, where the velocity magnitudes rise to about $1.7 \mathrm{~m} / \mathrm{d}$. After July 11,2015 , velocity magnitudes abruptly drop to $0.8 \mathrm{~m} / \mathrm{d}$. This observation coincides with the lake drainage and the associated glacier lake outburst flood [21]. This velocity temporal evolution is in agreement with [21]. Accordingly, the abrupt changes in velocity are successfully captured by the proposed method.

During the winter, the velocity magnitudes keep declining over the glacier tongue to reach $0.5 \mathrm{~m} / \mathrm{d}$ in April 2016. From 2016 to 2020, the velocity magnitudes appear to increase from March/April to June/July and drop from June/July to March/April (see also Figure 4). This shows the ability of the proposed method to retrieve a seasonal variability even though velocities have small magnitude (lower than $0.3 \mathrm{~m} / \mathrm{d}$ from 2016 to 2020).

Besides, in the lower part of the western tributary (zone B in Figure 6,7 and 8), the mean of velocity observation magnitude ranges from 0.1 to $10 \mathrm{~m} / \mathrm{d}$ ( $c f$. Figure 7). Such high velocities seem not realistic, considering the low surface slope between 2 and $5^{\circ}$ (cf. Figure 8). The mean data quality indicator of the velocity observations is about 0.1 in this zone. The noise in these velocity observations is due to a smooth surface covered by snow where features are hardly distinguishable. However, the leap frog velocity time series contains more values lower than $2 \mathrm{~m} / \mathrm{d}$ according to Figure 7 . Moreover, the difference between mean velocity magnitudes after and before inversion is about $-1.5 \mathrm{~m} / \mathrm{d}$ in zone B as illustrated in Figure 6 . The velocities after inversion are more consistent with the slope. Hence, the proposed method appears to decrease the noise even in areas where a few good quality displacement observations are available.

Finally, Figure 7 also highlights the interest of the method for providing a time series with a complete temporal coverage at a regular temporal sampling.

\section{CONCLUSION}

The described method allows for extraction of velocity time series with a regular and optimal temporal sampling using all available observations. The resulting time series have fewer gaps, no redundancy, and a complete temporal coverage. It relies on the temporal closure of the displacement observation network.

First, an improvement of the classical temporal closure formulation is proposed. The latter usually build time series 

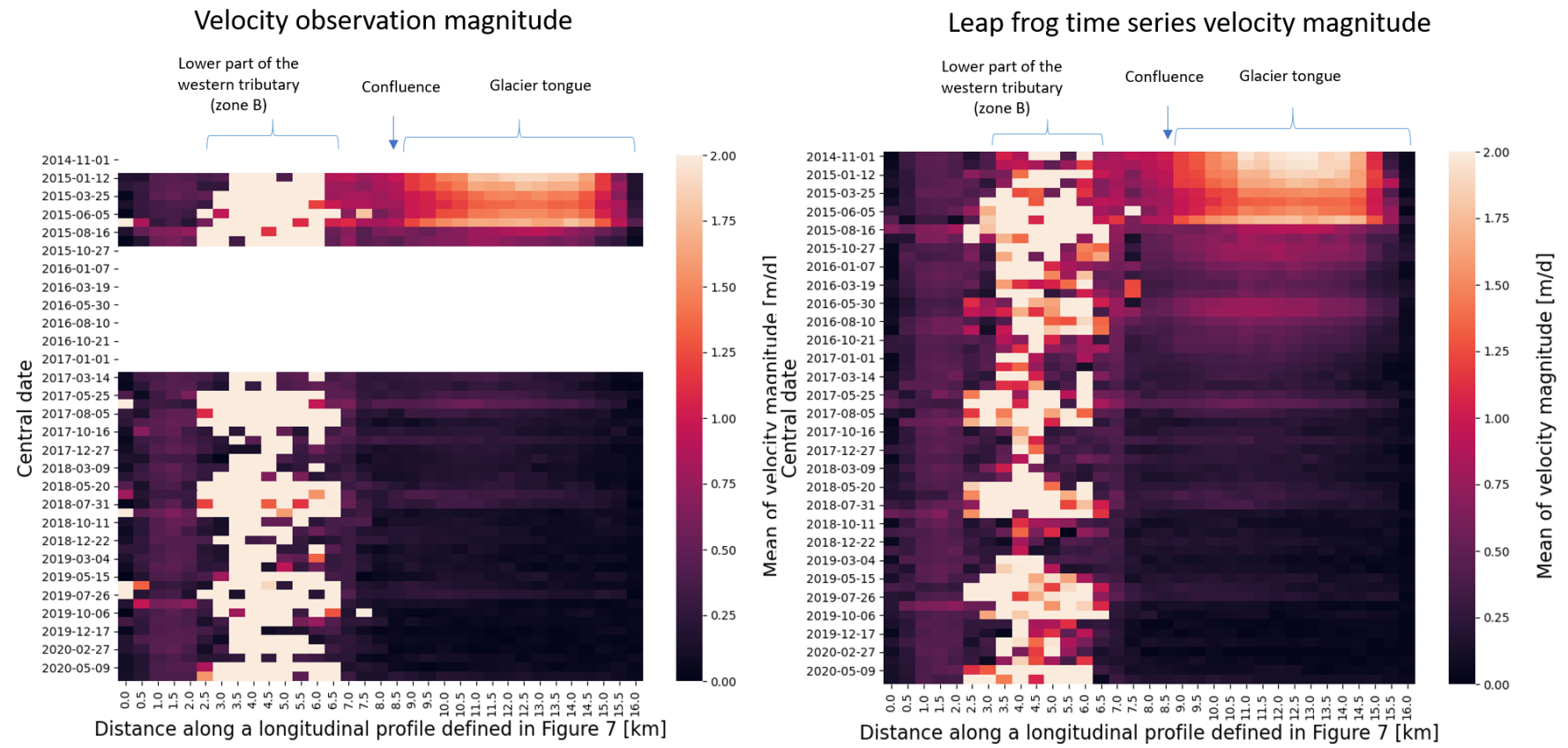

Fig. 7. Color-encoded matrix of velocity magnitude according to the central date of the velocity and the distance along a longitudinal profile represented in black in Figure 8. This longitudinal profile goes from the upper part of the western tributary to the glacier tongue. On the left, the color-encoded matrix stands for velocity observations with 36-days temporal baseline overlapping the 36-days sampling from the leap frog time series represented on the right. On the right, the color-encoded matrix represents leap frog velocity time series with a temporal sampling of 36 days.

with a temporal sampling equal to the satellite coverage frequency. However, since velocities are obtained through division of displacement by the temporal baseline, velocities obtained from a small temporal baseline can be significantly contaminated by noise. A new formulation of the temporal closure is proposed to obtain leap frog time series with a temporal sampling larger than the satellite coverage frequency from all available displacement observations. When a displacement observation can not be included in the classical temporal closure formulation because its start date or end date does not match the dates of the leap frog time series, a combination of displacement observations is used. Thereby, the proposed method enables building time series at a user-defined temporal sampling from all available displacement observations.

Second, the appropriate length of this temporal sampling to study short-term variations is discussed. Two criteria, the RMSE on stable ground and the VVC on moving areas, are proposed to analyze the signal-to-noise ratio of velocity time series with different temporal samplings.

Third, the data uncertainty is taken into account without the need for a preliminary data selection. Because the latter requires a reliable data quality indicator and a well-defined threshold value, in this article, an Iterative Reweighted Least Square is performed using the Tukey's biweight function. The internally-studentized residual resulting from this inversion is proposed as a proxy for the uncertainty.

Finally, the interest of the method is shown for mean velocities analysis. Since the resulting time series contain no more gaps and redundancy, each period has the same weight in the mean velocity computation.
A following up work will be to apply this method to other regions and data-set. This method could also be adapted to the case of landslide or slowslip displacements.

\section{ACKNOWLEDGMENT}

The authors would like to thanks Anna Derkacheva, Romain Millan and Jeremie Mouginot for their useful expertise concerning the management of ice velocity databases.

This research project benefits from the financial support of the French Ministry of Europe and Foreign Affairs, within the framework of the "Make Our Planet Great Again" Program for postdoctoral researchers.

\section{REFERENCES}

[1] A. S. Gardner, G. Moholdt, T. Scambos, M. Fahnstock, S. Ligtenberg, M. Van Den Broeke, and J. Nilsson, "Increased west antarctic and unchanged east antarctic ice discharge over the last 7 years," Cryosphere, vol. 12, no. 2, pp. 521-547, 2018.

[2] M. Fahnestock, T. Scambos, T. Moon, A. Gardner, T. Haran, and M. Klinger, "Rapid large-area mapping of ice flow using landsat 8," Remote Sensing of Environment, vol. 185, pp. 84-94, 2016.

[3] P. Friedl, M. Braun, T. Seehaus, S. Lippl, and P. Malz, "The retreatproject: derivation and distribution of remotely sensed variables for glaciers and ice caps outside of the polar ice sheets." in Geophysical Research Abstracts, vol. 21, 2019.

[4] R. Millan, J. Mouginot, A. Rabatel, S. Jeong, D. Cusicanqui, A. Derkacheva, and M. Chekki, "Mapping surface flow velocity of glaciers at regional scale using a multiple sensors approach," Remote Sensing, vol. 11, no. 21, p. 2498, 2019.

[5] A. Derkacheva, J. Mouginot, R. Millan, N. Maier, and F. GilletChaulet, "Data reduction using statistical and regression approaches for ice velocity derived by landsat- 8 , sentinel- 1 and sentinel-2," Remote Sensing, vol. 12, no. 12, p. 1935, 2020. 


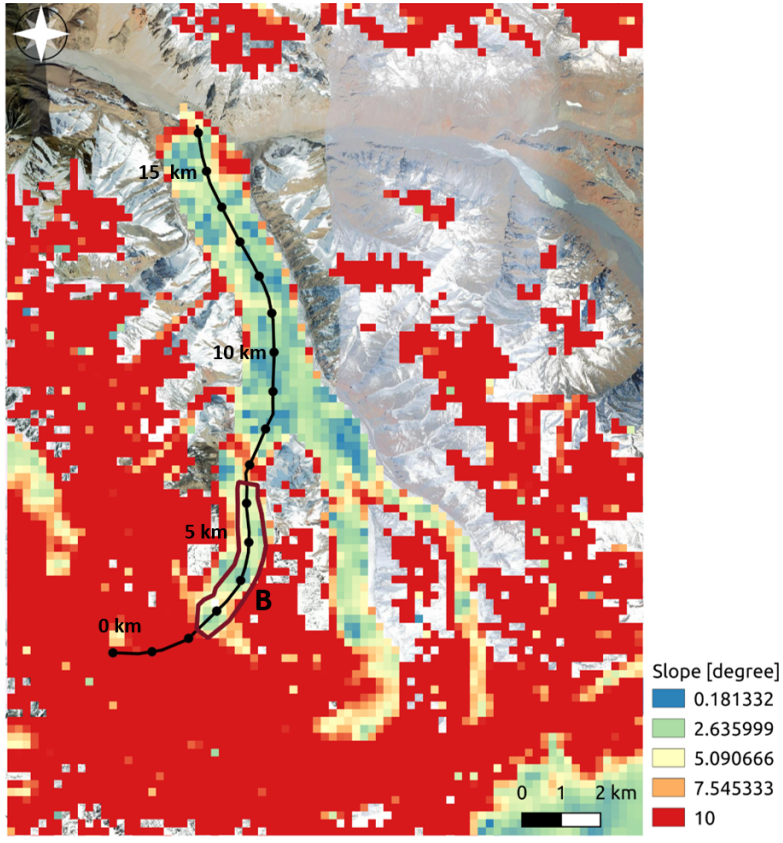

Fig. 8. Slopes computed using gdal from the Digital Elevation Model provided by [28]. This DEM has been wrapped with the projection EPSG 32643 and averaged with a window of $200 \mathrm{~m}$. On the regions not covered by glaciers according to the Randolph Inventory V6.0 [25] the slope are masked. These regions are instead represented with an optical image from the Google Earth collection. The longitudinal profile used in Figure 7 is represented in black with a black dot each $0.5 \mathrm{~km}$. Area B is encircled for analysis.

[6] A. Dehecq, N. Gourmelen, A. S. Gardner, F. Brun, D. Goldberg, P. W. Nienow, E. Berthier, C. Vincent, P. Wagnon, and E. Trouvé, "Twentyfirst century glacier slowdown driven by mass loss in high mountain asia," Nature Geoscience, vol. 12, no. 1, pp. 22-27, 2019.

[7] C. A. Greene, A. S. Gardner, and L. C. Andrews, "Detecting seasonal ice dynamics in satellite images," The Cryosphere, vol. 14, no. 12, pp. 4365-4378, 2020

[8] P. Berardino, G. Fornaro, R. Lanari, and E. Sansosti, "A new algorithm for surface deformation monitoring based on small baseline differential sar interferograms," IEEE Transactions on geoscience and remote sensing, vol. 40, no. 11, pp. 2375-2383, 2002.

[9] R. Lanari, F. Casu, M. Manzo, G. Zeni, P. Berardino, M. Manunta, and A. Pepe, "An overview of the small baseline subset algorithm: A dinsar technique for surface deformation analysis," in Deformation and Gravity Change: Indicators of Isostasy, Tectonics, Volcanism, and Climate Change. Springer, 2007, pp. 637-661.

[10] P. López-Quiroz, M.-P. Doin, F. Tupin, P. Briole, and J.-M. Nicolas, "Time series analysis of mexico city subsidence constrained by radar interferometry," Journal of Applied Geophysics, vol. 69, no. 1, pp. 115, 2009.

[11] F. Casu, A. Manconi, A. Pepe, and R. Lanari, "Deformation timeseries generation in areas characterized by large displacement dynamics: The sar amplitude pixel-offset sbas technique," IEEE Transactions on Geoscience and Remote Sensing, vol. 49, no. 7, pp. 2752-2763, 2011.

[12] L. D. Euillades, P. A. Euillades, N. C. Riveros, M. H. Masiokas, L. Ruiz, P. Pitte, S. Elefante, F. Casu, and S. Balbarani, "Detection of glaciers displacement time-series using sar," Remote sensing of Environment, vol. 184, pp. 188-198, 2016.

[13] L. Guo, J. Li, Z.-w. Li, L.-x. Wu, X. Li, J. Hu, H.-1. Li, H.-y. Li, Z.-1. Miao, and Z.-q. Li, "The surge of the hispar glacier, central karakoram: Sar 3-d flow velocity time series and thickness changes," Journal of Geophysical Research: Solid Earth, vol. 125, no. 7, p. e2019JB018945, 2020.

[14] N. Bontemps, P. Lacroix, and M.-P. Doin, "Inversion of deformation fields time-series from optical images, and application to the long term kinematics of slow-moving landslides in peru," Remote Sensing of Environment, vol. 210, pp. 144-158, 2018.

[15] H. Hadhri, F. Vernier, A. M. Atto, and E. Trouvé, "Time-lapse optical flow regularization for geophysical complex phenomena monitoring,"
ISPRS Journal of Photogrammetry and Remote Sensing, vol. 150, pp. 135-156, 2019.

[16] B. Altena, T. A. Scambos, M. Fahnestock, and A. Kääb, "Extracting recent short-term glacier velocity evolution over southern alaska and the yukon from a large collection of landsat data," The Cryosphere vol. 13, no. 3, pp. 795-814, 2019.

[17] K. Mason, Exploration of the Shaksgam Valley and Aghil Ranges, 1926. Asian Educational Services, 2004.

[18] Z. Xiangsong, "Investigation of glacier bursts of the yarkant river in xinjiang, china," Annals of Glaciology, vol. 16, pp. 135-139, 1992

[19] M. Zhang, F. Chen, B. Tian, D. Liang, and A. Yang, "Characterization of kyagar glacier and lake outburst floods in 2018 based on time-series sentinel-1a data," Water, vol. 12, no. 1, p. 184, 2020.

[20] C. Haemmig, M. Huss, H. Keusen, J. Hess, U. Wegmüller, Z. Ao, and W. Kulubayi, "Hazard assessment of glacial lake outburst floods from kyagar glacier, karakoram mountains, china," Annals of Glaciology, vol. 55, no. 66, pp. 34-44, 2014.

[21] V. Round, S. Leinss, M. Huss, C. Haemmig, and I. Hajnsek, "Surge dynamics and lake outbursts of kyagar glacier, karakoram," The Cryosphere, vol. 11, no. 2, pp. 723-739, 2017.

[22] H. Liang, L. Zhang, X. Ding, Z. Lu, X. Li, J. Hu, and S. Wu, "Suppression of coherence matrix bias for phase linking and ambiguity detection in mtinsar," IEEE Transactions on Geoscience and Remote Sensing, 2020.

[23] P. J. Huber, "Robust estimation of a location parameter," in Breakthroughs in statistics. Springer, 1992, pp. 492-518.

[24] Y. Yan, E. Trouvé, V. Pinel, G. Mauris, E. Pathier, and S. Galichet, "Fusion of d-insar and sub-pixel image correlation measurements for coseismic displacement field estimation: Application to the kashmir earthquake (2005)," International Journal of Image and Data Fusion, vol. 3, no. 1, pp. 71-92, 2012.

[25] R. Consortium et al., "Randolph glacier inventory-a dataset of global glacier outlines: Version 6.0," Global Land Ice Measurements from Space, Colorado, USA, Tech. Rep, 2017.

[26] A. Dehecq, N. Gourmelen, and E. Trouvé, "Deriving large-scale glacier velocities from a complete satellite archive: Application to the pamirkarakoram-himalaya," Remote Sensing of Environment, vol. 162, pp. 55-66, 2015.

[27] T. Nguyen, N. Méger, C. Rigotti, C. Pothier, E. Trouvé, N. Gourmelen, and J.-L. Mugnier, "A pattern-based method for handling confidence measures while mining satellite displacement field time series: Application to greenland ice sheet and alpine glaciers," IEEE Journal of Selected Topics in Applied Earth Observations and Remote Sensing, vol. 11, no. 11, pp. 4390-4402, 2018.

[28] D. Shean, "High mountain asia 8-meter dem mosaics derived from optical imagery, version 1.” 2017, boulder, Colorado USA. NASA National Snow and Ice Data Center Distributed Active Archive Center.

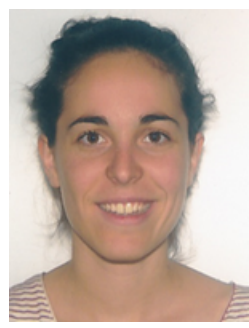

Laurane Charrier Laurane Charrier (Student Member, IEEE) received the M.S. degree in geological engineering from Ecole Nationale Supérieure de Géologie, Nancy, France in 2019 and the M.S. degree in remote sensing physics from University of Paris in 2019. She is pursuing a Ph.D. degree with LISTIC, Université Savoie Mont Blanc, Annecy, France and DTIS, ONERA, Paris, France. Her research interests include the estimation and fusion of displacement measurements for the study of glaciers. 


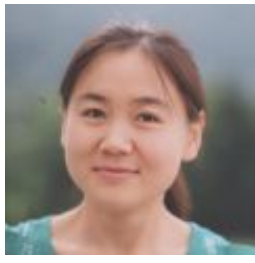

Yajing Yan Yajing Yan (Member, IEEE) received the M.S. degree in Fundamentals of remote sensing from University Paris 6, Paris, France, in 2008, and the Ph.D. degree in geosciences and environment from LISTIC and Institut des Sciences de la Terre (ISTerre), University Savoie MontBlanc, Annecy, France, in 2011. She was a PostDoctoral Fellow with Institut d'Électronique et des Télécommunications de Rennes (IETR), University Rennes 1, Rennes, France, from January 2012 to June 2012 and with Geo-Hydrodynamics and Environment Research of University of Liège, Liège, Belgium, from July 2012 to August 2014. Since September 2014, she has been an Associate Professor with LISTIC, University Savoie Mont-Blanc. Her research interests include multitemporal InSAR processing, data fusion and data assimilation.

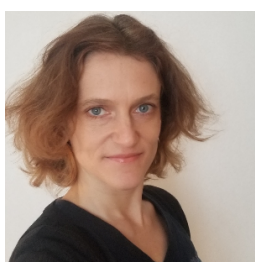

Elise Colin Koeniguer Elise Colin Koeniguer received jointly the Dipl. Ing. in electrical engineering from Supelec and the M.Sc. degree in theoretical physics, from the University of Orsay (Paris XI), Paris, France, in 2002. In 2005, she received a $\mathrm{Ph} . \mathrm{D}$. degree from the University of Paris VI, Paris, France, and joined the Electromagnetic and Radar Division of ONERA, Palaiseau, France, in which she has been working on the comparison between radar polarimetry and optical polarimetry. In 2013, she joined the Information Processing Department and obtained the Habilitation à Diriger des Recherches from the University ParisSud, Orsay, France, in 2014. She leads several research projects in remote sensing, especially on AI, big data, coherent images, and time series. She is also a co-founder of ITAE medical Research, a company developing a vascular imaging device based on dynamic speckle and polarimetric properties of light.

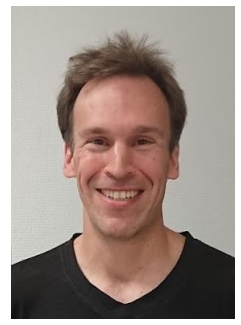

Sivain Leinss Silvan Leinss (Senior Member, IEEE) received the Dipl. degree in laser physics from the University of Constance, Konstanz, Germany, in 2008 and the Ph.D. degree in radar remote sensing from ETH Zurich, Zurich, Switzerland, in 2016. In 2009, he was working in the field of coherent light scattering at the University of Constance and in 2010, in the field of light scattering statistics in Bose-Einstein-Condensates at ETH Zurich. Since 2021, he has been with the Laboratoire d'Informatique, Systémes, Traitement de l'Information et de la Connaissance (LISTIC), Polytech Annecy-Chambéry, Université de Savoie Mont Blanc,Chambéry, France. Dr. Leinss was the recipient of the Helmut-Rott-Prize for his excellent contributions for remote sensing of the cryosphere.

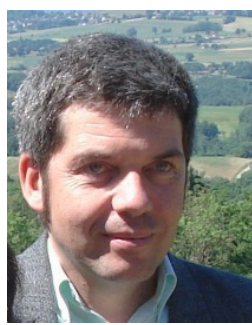

Emmanuel Trouvé Emmanuel Trouvé (Senior Member, IEEE) received the Engineer degree in electrical engineering from the École Nationale Supérieure de Techniques Avancées, Paris, France, in 1990, the Ph.D. degree in signal and image processing from Ecole Nationale Supérieure des Télécommunications, Paris, France, in 1996, and the "Habilitation à Diriger des Recherches" degree from Université of Savoie, Chambéry, France, in 2006. From 1996 to 1998, he worked with Thomson Marconi Sonar in Underwater Acoustic and Signal Processing. He has been working with University Savoie Mont Blanc, Polytech Annecy-Chambéry, Laboratoire d'Informatique, Systèmes, Traitement de l'Information et de la Connaissance, as an Associate Professor (1998-2008), then as a Professor of signal and image processing. His research interests include synthetic aperture radar (SAR) image processing, photogrammetry and glacier monitoring by remote sensing. Prof. Trouvé has been coordinating several research projects, including the national multilaboratory project EFIDIR (Extraction and Fusion of Information for Displacement measurement from SAR Imagery, 2008-2012). He was the General Chair of MultiTemp 2015 (8th International Workshop on the Analysis of Multitemporal Remote Sensing Images) and the treasurer of the French Chapter of the IEEE Geoscience and Remote Sensing Society from 2013 to 2016 . 